\title{
Le chef et la pluie chez les Mofu, montagnards du Nord-Cameroun
}

(Contribution à l'étude des formes du pouvoir politique)

Chiefs and rain among the Mofu, mountaineers in northern Cameroun: A contribution to the study of forms of political power

Jeanne-Françoise Vincent

\section{OpenEdition}

\section{Journals}

Édition électronique

URL : http://journals.openedition.org/span/232

DOI : $10.4000 /$ span.232

ISSN : 2268-1558

Éditeur

École pratique des hautes études. Sciences humaines

Édition imprimée

Date de publication : 1 septembre 1975

Pagination : 137-164

ISSN : 0294-7080

\section{Référence électronique}

Jeanne-Françoise Vincent, "Le chef et la pluie chez les Mofu, montagnards du Nord-Cameroun », Systèmes de pensée en Afrique noire [En ligne], 1 | 1975, mis en ligne le 08 juillet 2013, consulté le 30 avril 2019. URL : http://journals.openedition.org/span/232 ; DOI : 10.4000/span.232 
LE CHEF ET LA PLUIE CHEZ LES MOFU, MONTAGNARDS DU NORD-CAMEROUN

(Contribution à l'étude des formes du pouvoir politique)

par

Jeanne-Françoise VINCENT

PROPOS : Montrer comment, chez les Mofu, le pouvoir politique possède nécessairement un aspect magique : on reconnait au chef de massif le privilège d'attirer ou de retenir les pluies au dessus de ses montagnes, voire de celles des voisins.

RESUME : Les massifs mofu ne possèdent pas une organisation politique identique. Dans huit de ces massifs l'autorité est émiettée entre les divers chefs de quartiers. Dans les trois derniers, au contraire, il existe un seul chef pour tout le massif.

Les uns et les autres, cependant, lient de la même façon le pouvoir sur la pluie à la manipulation de pierres spéciales, les unes donnant la pluie, les autres l'empêchant. Les pierres à pluie sont conçues comme des sortes de poissons; au contraire les pierres arrêtant la pluie sont liées à l'arc-enciel, créateur de sécheresse, et posséderaient un pouvoir beaucoup plus difficile à manier. Les premières sont manipulées au cours de sacrifices aux ancêtres, et accessoirement aux génies de la montagne. Les secondes appartiennent par contre à un domaine beaucoup plus magique que religieux.

L'examen des mythes de peuplement des massifs montre que, pour les Mofu, la dëtention des pierres de piuie est liée à l'exercice de l'autorité. Un chef véritable doit en posséder, dût-il les acquérir par la ruse ou la violence. Toutefois, ce pouvoir sur les pluies n'est pas considéré comme forcement bénéfique et si une sécheresse se produit, le chef en est tenu pour responsable. On aperçoit donc l'ambiguité de ce pouvoir qui suivant lescas apparalt comme le couronnement de l'autorité ou comme la faille permettant de la contester.

"L'eau donne naissance à tout : au mil, aux arbres, aux personnes aussi, puisque l'eau chaude sert à nourrir le petit enfant. Les betes aussi ont besoin de l'eau. Notre vie vient de l'eau ; aussi c'est à cause de la pluie que l'on craint vraiment un chef". Voilà ce que me disait Ndekelec, un vieux Mofu du massif de Durum en 1973, lors de ma dernière mission, au cours d'une conversation où je l'interrogeais sur l'histoire de son massif et sur les chefs qui s'étaient succédés à sa tête. 
Rien de plus banal que le début de cette remarque : oui, pour des cultivateurs de sahel, l'eau c'est la vie, mais ce qui l'est moins, c'est le lien aussitôt affirmé entre le chef et la pluie : c'est le chef qui apporte la pluie sur son massif ; c'est son pouvoir sur la pluie, manifesté chaque année, qui assied sor autorité. En fait, ce pouvoir n'est qu'une des composantes de son autorité, aussi n'est-il pas inutile, après une brève présentation des Mofu et de leur milieu physique, de donner quelques explications sur le fonctionnement de la chefferie mofu. Ensuite seulement, j'expliquerai à l'aide de quoi se manifeste le pouvoir du chef sur la pluie, en exposant les idées mofu sur l'existence de "pierres de pluie", pierres pour l'attirer et pierres pour l'arrêter. Puis je montrerai comment apparait le lien entre chef et pierres de pluie, au niveau du mythe et au niveau du rite.

Cet exposé sera surtout descriptif, simple mise en forme de notes de terrain, certaines très récentes, donc pas encore totalement assimilées.

I - PRESENTATION DES MOFU.

Les Mofu occupent une région de montagnes de moyenne altitude (700 à 1000 mètres). Ils sont installés sur le rebord oriental des Monts du Mandara, ainsi qu'autour de montagnesîles, dispersées à travers la plaine du Diamaré. Sauf à proximité des montagnes-Iles, les Mofu sont encore peu ou pas descendus en plaine. Ce sont donc de vrais montagnards. Leur: pays se trouve un peu au sud du IIe parallèle. La hauteur des pluies s'y amenuise d'ouest en est, et du sud au nord. Elle va de 850 à 950 mns par an jusqu'à 600 à $700 \mathrm{mms}$. Le volume des pluies parait donc suffisant : en fait, il peut être très mal réparti.

Les Mofu cultivent presqu'uniquement le mil, mil de montagne surtout (puisque, rappelons-le, l'habitat de montagne est encore généralisé) et, tout récemment, mil repiqué de plaine emprunté aux Peul voisins. Les cultures d'exportation, arachide et coton sont, elles aussi, très récentes et ne peuvent concurrencer le mil. 
On compte environ 40 000 Mofu répartis sur 11 massifs. Parmi ces massifs, on peut distinguer trois ensembles, deux dont les liens sont assez láches - car constitués essentiellement par l'intégration à un même cycle de fêtes religieuses et ne présentant pas de langue commune. Ce sont les groupes de massifs de Zulgo - Gemžek - Meri - Mboko (16 Ooo personnes environ) et Molkwo - Tšere - Mikiri - Dugwor (11.000 personnes environ), où sont parlées 6 langues différentes.

Un troisième groupe, formé des massifs de Duvangar, Durum et Wazan (représentant 13000 personnes environ) est beaucoup plus homogène. La même langue $y$ est parlée et les mariages sont pratiqués presqu'exclusivement entre les trois massifs (1). Comme dans les deux autres groupes de massifs, c'est la célébration des fêtes religieuses qui crée la parenté (entre ces massifs) mais là, en plus, on trouve un système social et religieux rigoureusement superposable d'un massif à l'autre.

Les Mofu ont pour voisins proches à la fois des montagnards et des gens des plaines. Au nord, ils sont en contacts immédiats ou non, avec 5 groupes montagnards peu importants numériquement (de 6 à 15 ooo personnes) : les Mada, Muyan, Muktele, uldeme et Podokwo. A l'ouest, ils jouxtent le plus gros groupe ethnique montagnard, les Matakam ou Mafa (120 ooo environ). Au sud, ils touchent les Gudur (16 000 environ), longtemps confondus avec eux.

Leurs voisins des plaines se trouvent à l'est de leurs massifs. Les plus proches sont les Giziga (55 0oo environ) divisés en plusieurs chefferies importantes, celles de Marva et de Kaliao étant en contact direct avec eux. Les Peul (270 000 pour tout le Nord-Cameroun) ont eu avec les Mofu dans le passé des rapports belliqueux, constitués essentiellement de razzias, qui les ont fortement marqués, les deux chefferies peul auxquelles ils se sont heurtés étant celles de Maroua et Gazawa.

(1) J.F. Vincent: "Données sur le mariaqe et la situation de la femme mofu, (massifs de Duvangar et de Wazan, Cameroun du Nord)", Cah. Orstom, Ser. Sc. Hum. Vol. IX $n^{\circ} 3,1972$, pp. 309-323; cf. p. 314 . 
Au nord des Peul, le petit royaume noir du Mandara a été également en relations avec les massifs mofu, relations de conquête et de violence pour les massifs les plus exposés, mais relations pacifiques car basées sur le commerce avec les massifs les plus éloignés.

II - FONCTIONNEMENT DE LA CHEFFERIE.

1) distinction massifs-quartiers.

Il faut d'abord faire une distinction importante au niveau du vocabulaire, en expliquant ce qu'on entend par "massif" et par "quartier".

Les observateurs des différentes ethnies montagnardes du Nord-Cameroun soulignent leur division en "massifs". Je viens également d'employer plusieurs fois le terme. Ce terme est ambigu car il possède deux sens. Le premier est un sens géographique précis. "Massif" désigne alors un ensemble montagneux plus ou moins vaste, le cas échéant avec sa zone de piémont, possédant des limites nettes, vallées ou plaines, et portant un nom d'ensemble. Par exemple le massif de Wazan, Ngwa ma wazan,chez les Mofu, le massif de Baldama chez les Muktele(3) le massif de Magumaz chez les Mafa (4). Cet ensemble est facile à individualiser dans le cas des montagnes-îles. Assez souvent pourtant, les limites du massif paraîssent géographiquement arbitraires, surtout lorsque ce "massif" est en fait une portion de plateau montagneux. Pourquoi choisir pour limite telle vallée plutôt que telle autre ? C'est bien ce que l'on se dit en considérant les limites entre les massifs de Meri et celui de Duvangar par exemple, ou encore celles entre le massif de Duvangar et celui de Durum. Il faut, en fait, faire référence au groupe humain occupant ce terroir.

(2) J.F. Vincent: "Eléments d'histoire des Mofu, montaqnards du Nord-Cameroun". Contribution au Colloque CNRS de sept. 1973 sur 1 'ethno-histoire du Cameroun. Doc. multigr. 52 p., 2 c., à paraltre dans les Actes du Colloque en 1976.

(3) B. Juillerat: Les bases de Z'organisation sociale chez les Mouktele (Nord-Cameroun), structures lignagères et mariage Far'is, Mém. Inst. Ethnol, VIII, 1971, 271 p.

(4) J.Y. Martin: Les Matakam du Cameroun $\mathrm{n}^{\circ} 41,1970,215 \mathrm{p}$.

Paris, Mém. Orstom, 
Car le mot "massif" possède aussi un sens sociologique et désigne alors non plus le relief mais le groupe. "Massif" signifie en ce cas unité politico-religieuse pouvant ou non reconnaitre l'autorité d'un seul chef. C'est pris en ce sens que le massif se subdivise en "quartiers", que l'administration appelle parfois "villages", en particulier dans les listes de recensement. Un quartier possède lui aussi des limites géographiques. Au départ, il a été occupé par un seul clan et présentait donc une homogénéité classique. Cependant, cette homogénéité classique a été rarement conservée ; par le jeu des minimigrations à l'intérieur du massif, elle s'est transformée progressivement en hétérogénéité. Toutefois, le clan fondateur garde souvent un statut privilégié l'amenant alors à fournir le chef du quartier.

\section{2) dévolution de l'autorité.}

Dans 8 massifs sur 1I, du massif de zulgo à celui de Dugwor, on trouve une autorité émiettêe et il n'y a pas de chef pour I'ensemble du massif. On peut parler à ce niveau d'"an-archie", puisqu'il y a absence d'un chef de groupe. Le seul pouvoir reconnu s'exerce à l'échelon du quartier. Géographiquement restreint, il est pourtant assez diversifié.

Ce pouvoir est essentiellement religieux : le chef de quartier est le desservant d'un culte adressé à l'esprit de la montagne protégeant l'ensemble du quartier, qui se trouve donc unifié sur le plan religieux. C'est lui aussi qui donne le signal des grandes fêtes célébrées par tout le quartier le même jour,fêtes annuelles et fêtes triennales ou quadriennales qui fondent la cohésion sociale du groupe.

Le chef de quartier a aussi un pouvoir économique. Il est le martre des cultures. C'est sur son ordre que débutent les travaux des champs, en particulier le nettoyage avant les semailles. Il a droit à de modestes prestations en travail, effectuées par la promotion de jeunes gens du quartier, une fois tous les trois ou quatre ans.

Ce pouvoir du chef de quartier est enfin judiciaire, ou 
plutôt il lui permet de jouer les conciliateurs en cas de conflits mais aucune sanction n'est prévue.

Dans ces 8 massifs mofu, il y a sur le même massif coexistence de plusieurs chefs de quartier dont aucun ne s'est imposé aux autres. Au contraire, dans les 3 massifs restants, Duvangar, Durum et Wazan, on rencontre une concentration de l'autorité. Cette fois, il s'agit de véritables chefferies dont les limites coincident avec celles du massif géographique. Là aussi pourtant, il y a division en quartiers, mais ces quartiers reconnaissent tous l'autorité d'un même chef de massif. Le chef.de quartier perd son rôle économique et judiciaire, ainsi qu'une partie de son rôle religieux : il ne donne plus le signal des fêtes et $n$ 'est que desservant d'un culte de quartier. Pour tout le reste, il est "dans la main" du chef de massif. Tolitefois, les mythes de peuplement montrent bien que cette dépossession s'est faite par étapes.

Dans sa nature, le pouvoir du chef de massif n'est pas différent de celui du chef de quartier ; il est à la fois religieux et économique; (on ne rencontre pas chez les Mofu de distinction entre le chef politique et un "maitre de la terre" dont le rôle serait plus nettement religieux). Toutefois, les avantages économiques du chef de massif sont beaucoup plus importants : il a droit à des corvées régulières, à des "plantations du chef" dans chaque quartier et à des redevances en bière de mil et en viande. De plus, il a le droit de prendre chèvre, fille, et parfois plantation.

Ce pouvoir s'exerce aussi sur un champ géographique plus vaste puisqu'il s'étend au massif. Mais il ne va pas au delà. Sur les trois massifs, on trouve trois chefferies construites sur le même modèle rnais aucune n'a essayé d'absorber les autres.

Le pouvoir du chef de massif s'exerce en un plus grand nombre de domaines que celui du chef de quartier. Le chef de massif joue d'abord un rôle socio-économique important puisqu'il est à la fois maître de la terre cultivée qu'en certains cas il peut redistribuer (sans droits sur la brousse, chasse 
et peche etant presque inexistantes), maitre des cultures et des travaux des champs, maltre de la guerre et de la paix.

Il possède également un rôle religieux très proche de celui du chef de quartier, étant a la fois desservant d'un génie de la montagne - présenté comme le plus grand et etendant sa protection a tout le massif cette fois - et maitre des sacrifices : seul, il donne le signal des grandes fêtes.

Le chef de massif joue aussi un rôle judiciaire qui peut en certaines circonstances apparaitre comme apparenté à son role religieux : c'est ainsi que lui seul arbitre les ordalies et serments solennels en cas d'accusation grave. Par ailleurs, lorsqu'il juge des cas simples, le chef de massif peut prononcer des sanctions, en particulier percevoir des amendes; 11 dispose ainsi d'un pouvoir judiciaire réel.

Le rôle du chef de massif présente enfin un aspect magico-religieux. On peut meme parler d'un pouvoir mystique du chef qui est cense lutter contre les sorciers et les epidemies avec I'aide de voyants, mais sans être voyant lui-même.

C'est à cet aspect magico-religieux que se rattache le pouvoir que le chef de massif possede, dit-on, sur les pluies, pouvoir qu'il ne peut partager avec personne d'autre sur l'étendue de son massif. A la différence du chef de quartier qu土, dans les massifs à autorite émietté, peut ou non avoir pouvoir sur la pluie, sur les trois massifs de Duvangar, Durum et Wazan, le chef de massif doit necessairement controler les pluies. L'etude des mythes de peuplement montrera toutefois que ce pouvoir sur les pluies n'a pas eté dès les origines l'apanage du clan detenant aujourd'hui la chefferie.

III - RELATIONS ENTRE LE CHEF ET LA PLUIE.

1) Les pierres de pluie.

Pour les Mofu, le pouvoir sur la pluie est lié à la manipulation de pierres de plule dont on m'a beaucoup parle et que j'ai pu observer par moi-meme, mais a de rares occasfons: 
Je m'appuierai donc surtout sur des descriptions théoriques.

Selon les Mofu, il existe deux sortes de pierres de pluies : celle qui font venir la pluie ou bizi yam, expression qui peut se traduire et signifie "enfants de la pluie" et celles qui l'arretent, les kwalay, mot rendu parfois par "arc-enciel".

a) les pierres pour faire venir la pluie : les bizi yam ou "enfants de la pluie". (Ici, il s'agit de l'appellation en langue de Duvangar-Durum-Wazan; mais chaque langue a bien sar la sienne, biza wra, en langue de Molkwo par exemple), On les découvrait souvent autrefois, dit-on : ces bizi yam consti-. tuaient alors une sorte de collection en extension continue. Il semble que maintenant, ces découvertes se fassent plus rares. Ainsi, le chef de pluie de Morley, qui est chef de pluie depuis huit ans, m'a dit qu'on ne lui en avait encore apporté. aucune.

Seul le hasard préside à leur découverte; on ne part pas spécialement à leur recherche. On les trouve presque toujours à l'intérieur de quelque chose, généralement dans la terre, en recueillant de l'argile, en creusant des trous à côté de puits, ou encore en faisant la chasse aux rats des champs et en agrandissant leur terrier. Dans tous ces cas, les pierres seraient placées dans des sortes de "nids", faits de brins d'herbe. Parfois, lorsque le tonnerre est tombé, on découvre qu'il a fendu des rochers à l'intérieur desquels on trouve également des pierres de pluies, présentées alors comme "tombées du ciel" avec la foudre.

Les "enfants de la pluie" sont des pierres arrondies, "comme des oeufs", suivant une comparaison fréquente. Les plus courantes sont de cette taille mais il y en a aussi de grandes dimensions. Pourquoi "arrondies" ? Parce que - je les ai vues à quatre reprises - il s'agit d'outils néolithiques de pierre polie et également de percuteurs hors d'usage, raviveurs à mortier dont l'ancien usage est totalement insoupçonné. Il m'a été dit qu'il fallait "des pierres de plusieurs sortes" : des longues, des rondes, des plates, etc... Elles sont gardées toutes ensemble dans une poterie soigneusement recouverte dont elles ne sortent que pour être manipulées lors des rites de pluie. 


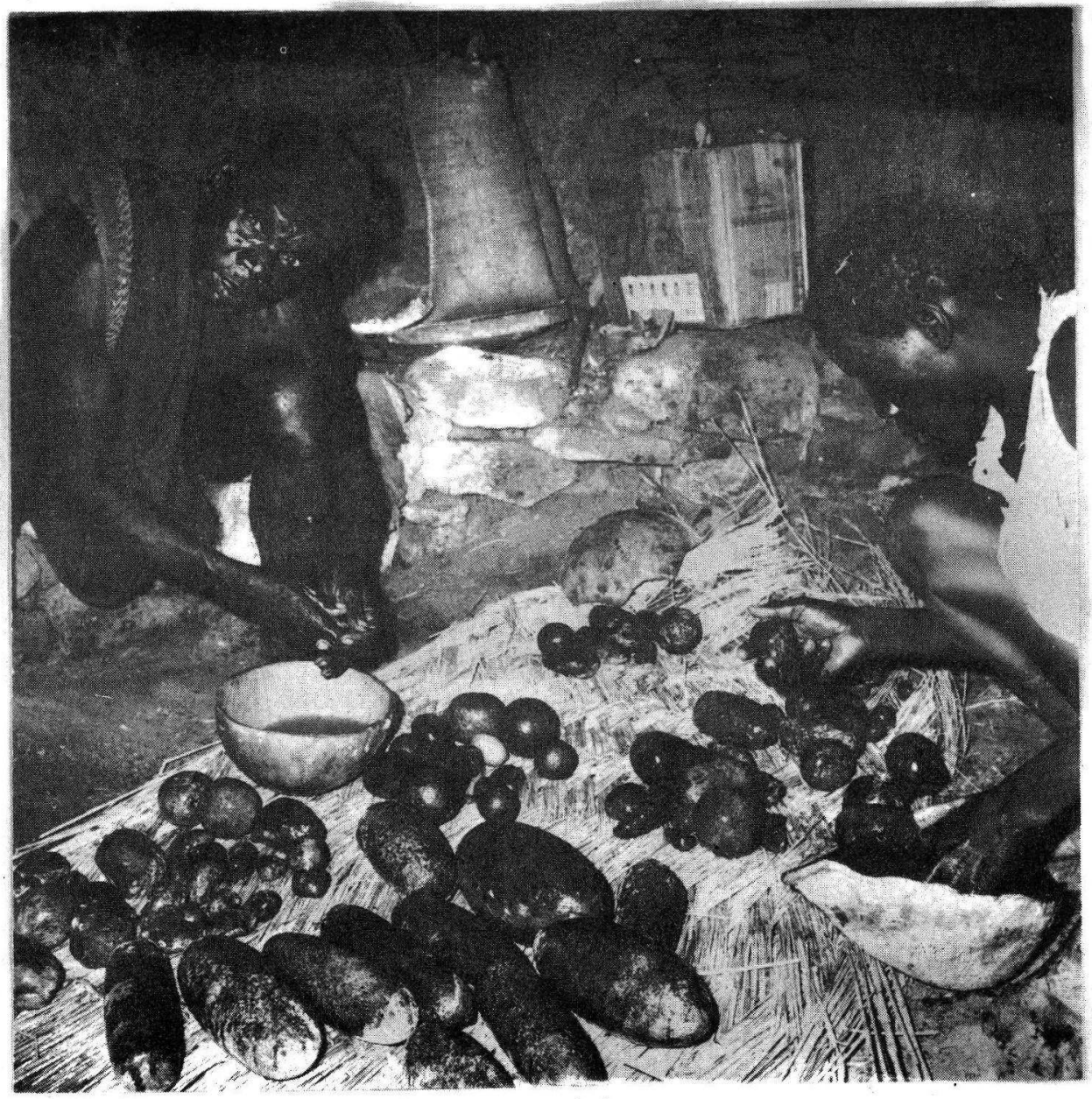

- Les "enfants de la pluie" sont lavés au cours d'un sacrifice de demande de pluie. Wazan (Nord-Cameroun), mai 1969. 
"Le vrai bizi yam, lorsqu'on le met dans sa main et qu'on souffle dessous, il vient à cet endroit comme de l'eau". Cette précision insiste sur l'humidité supposée des pierres de pluie. Cette humidité ne se rencontre pas chez toutes, mais il y a au moins une des pierres de la collection qui reste continuellement humide. Il ne faut donc pas qu'elles voient le soleil pendant la saison des pluies ; cela tarirait leur pouvoir.

On trouve aussi l'idée de la présence d'une force dans chaque pierre; "chacune contient comme un esprit". D'où l'inquiétude du chef de pluie de Morley dont la case avait brolé avec les pierres de pluie. "Avaient-elles encore leur pouvoir ?". Elles ont une sorte de vie qui leur permet de se déplacer; on raconte qu'elles peuvent alors brouter l'herbe. Si celui qui la trouve ne plaît pas à la pierre, si "elle ne l'aime pas", elle disparait toute seule de chez lui. Selon certains, cette vie des pierres de pluies ne se manifesterait pas en saison sèche. Un informateur m'a dit: "quand la pluie tombe, les "enfants de la pluie" deviennent comme des poissons; si la pluie vient sur eux, ils se mettent à glisser les uns sur les autres et à frétiller ; ils peuvent alors se promener dans l'eau". Selon un autre, lors des sacrifices de demande de pluie, les bizi yam peuvent flotter dans l'eau avec laquelle ils sont lavés. Ces pierres peuvent vieillir et ellès finissent par "mourir". Dans une collection de pierres de pluie, il y a donc des "mortes" et des "vivantes". On expérimente leur pouvoir en les mettant séparément dans $l^{\prime}$ eau : on détermine ainsi celles qui sont longues à faire venir la pluie ou qui ne la font plus venir. On se débarasse des plus vieilies en les enterrant avec les chefs.

Leur vie, leur force, les rendent dangereuses à manier. S'il est un homme ordinaire, leur "inventeur" ne doit pas les garder, sinon il sera tué par la foudre. Seul un individu habilité, le chef de pluie, peut les posséder. Souvent, il donnait en échange un boubou ou des chèvres au rabatteur. Sur le massif de Molkwo, on chuchote que le chef aurait achete 5000 FCFA une pierre de pluie. Cette somme énorme - l'impôt annuel tourne autour de 1000 FCFA par homme adulte - montre bien quelle valeur leur est attachée. 
Les pierres à pluie sont gardées dans la maison du chef, dans un de ses greniers ou dans sa propre chambre à coucher. Seul le chef peut les manier, car il peut neutraliser leur côté dangereux par des remèdes magiques appropriés à base d'herbes. En dehors de lui, deux personnes peuvent les toucher, un serviteur et un notable, tous deux nommés à vie par lui.

Leur nombre est très variable : chez le chef de wazan j'en ai vu près de 80 à la fois ; par contre le chef de Morley n'en avait que 2. Il ne semble pas que le pouvoir du chef de pluie soit lié à leur grand nombre, car le chef de Morley est dit contrôler les pluies avec plus d'efficacité que celui de Wazan.

Les "enfants de la pluie" changent de place suivant les saisons; rangés en saison sèche, ils sont sortis en saison des pluies.

b) les kwalay ou "pierres empêcheuses de pluie".

Le kwalay a été parfois assimilé à l"arc-en-ciel", sans préciser qu'il s'agissait d'une traduction ou d'une comparaison. En tout cas, c'est le même mot qui est employé sur les 11 massifs mofu (5)

A la différence des "enfants de la pluie", les kwazay sont toujours en nombre très restreint, un, parfois deux par chef, et on se rappelle les circonstances de leur acquisition. La découverte d'un kwalay est fortuite, le plus souvent à la suite d'une grande sécheresse. Il arrive aussi que le kwalay ait été acheté à des étrangers au massif ; tel le kwalay de Wazan, dont on connait encore le nom, le clan et le massif du vendeur. A Molkwo, on indique même le montant de l'achat effectué là aussi auprès d'étrangers - 8 boeufs, 3 "pièces d'argent" (probablement 3 thalers) et 3 boubous de coton.

(5) On retrouve ce terme de kwalay chez les Gudur, ainsi que je l'ai constaté lors d'une enquête comparative. Quant aux Giziga qui utilisent, eux aussi, les deux types de pierres de pluie, ils emploient le mot kolay pour désigner la pierre provoquant l'arrêt des pluies; cf. J. Fourneau: "Une tribu païenne du NordCameroun, les Guissiga (Moutourova) contribution ethnographique" in Journal de la Société des Africanistes, VIII-2, pp. 163-195, 1938 . 
Le kwalay est une pierre arrondie, multicolore, avec du rouge et du jaune, parfois du vert, (d'où l'appel à l'arc-enciel). Ces couleurs font"comme des veines". Le rouge domine sans doute car parfois on en parle comme d'une pierre rouge. On peut se faire une idée de leur apparence supposée en se rappelant qu'il y a quatre ou cinq ans, furent vendues sur le marché de Maroua des balles de plastique transparent, très dures, avec des veines de couleurs, semblables à de grosses billes d'agathe. L'une d'elles arriva jusque sur le massif de Duvangar et provoqua la perplexité des vieux qui, après l'avoir bien retournée, la rendirent aux enfants en concluant qu'il ne s'agissait pas d'un kwalay. Avec les idées sur le kwalay, on aborde un domaine nettement plus magique que celui des"pierres de pluie" car le kwalay est présenté expressément comme un être vivant, et même comme une personne, ndo, "une toute petite personne", qui peut se déplacer. Un informateur m'a dit: "il est vivant, il marche; il se promène lui aussi, mais pas en roulant, en glissant". A mes questions, il a précisé: "tout de même, il n'a ni yeux, ni pattes".

Il est présenté comme vivant au fond d'un trou,d'où il sort parfois pour "manger les nuages". On dit qu'il peut voler dans les airs et que la pluie s'arrête. Une fois sorti, il part en brousse et se perche dans certains arbres oũ il faut essayer de le retrouver. Il s'agit alors d'une quête mystique et non pas réelle : le kwalay se sauve "en esprit", peut-on dire. Le devin qui dirige cette quête peut seul voir à quel moment le kwalay est rattrapé.

Ces croyances sur le kwalay vivant au fond d'un trou et apportant la sécheresse par sa sortie, expliquent que le grand souci d'un chef de pluie soit d'enfermer convenablement son kwalay. Il est mis dans plusieurs jarres, parfois jusqu'à 6, où il peut être enveloppé au départ dans une peau de chèvre; ces jarres s'emboitent les unes dans les autres et sont placées dans une fosse très profonde recouverte de terre bien tassée. Parfois, des barres de fer sont mises en travers. 
En principe, le kwalay ne sort jamdis de cette fosse. "Une fois enterré, il n'est plus touchē". Presqu'à la surface de la fosse, on dispose une couche d'ocre rouge recouverte de terre ordinaire. Chaque année, on scrute la surface de la fosse. Si l'on y aperçoit des trous, on les interprète comme une demande du kwalay. "Le kwalay est fâché ; il cherche à sortir". Pour l'en empêcher, on lui sacrifie une chèvre rouge.

Ces deux sortes de pierres, "pierres à pluie" et "empecheur de pluie" doivent aller de conserve. Cette obligation m'a été soulignée à diverses reprises. "Un vrai chef de pluie doit avoir les deux pouvoirs ; un seul ne sert à rien".

\section{2) Pierres de pluie et chefferie dans les mythes.}

Dans les deux types de mythes que l'on rencontre chez les Mofu : mythes d'origine et mythes de peuplement, on trouve des allusions aux pierres de pluie, particulièrement répandues dans les seconds.

Parmi les mythes d:origine, ceux qui concernent le feu, la mort, voire les débuts du monde et de l'espèce humaine, sont peu répandus et peu détaillés; les rares personnes les connaissant sont toujours des gens âgés. Par contre, le mythe sur l'origine du mil est très populaire (6) et m'a été conté, parfois très longuement, par des informateurs de tous âges, hommes et femmes. Selon le mythe, il existait autrefois un mil extraordinaire dont un quart de grain suffisait à nourrir une famille. Ce mil, par la faute d'une femme, devint le mil banal actuel. Il avait été trouvé au ciel oũ il avait été dérobé à Dieu par un homme appartenant au clan Medey, appelé encore Movo, à qui sont toujours attribués divers pouvoirs magiques sur les maladies par exemple, et aussi sur les sauterelles ou les panthères qu'ils peuvent aussi bien combattre qu'envoyer. Une seule fois, $j$ 'ai recueilli une variante expliquant qu'en même temps que le mil avaient été dérobés un kwaíay et un bizi yam. L'intérêt de cette variante est de lier expressément

(6) Il déborde d'ailleurs l'aire mofu ; je l'ai retrouvé chez les Gudur et chez les Mafa. 
le début du pouvoir sur la pluie au clan Medey, dont des représentants existent sur presque tous les massifs Mofu, ou ils sont soumis à l'autorité d'autres chefs. Cependant, en un cas, à Morley, quartier qui relève aujourd'hui administrativement de Wazan, ils constituaient une minuscule chefferie jadis indépendante, un "massif" dont le chef est toujours considéré comme le plus redoutable faiseur de pluie de toute la région.

Dans le second type de mythes, les mythes de peuplement, les pierres de pluie apparaissent constamment et sont toujours liées à la chefferie, de quartier ou de massif. Fautil vraiment parler de mythes, à propos de ces récits racontant comment les divers clans d'un même massif $y$ ont fait leur apparition et comment ils ont fusionné peu à peu en un même groupe ? Oui, car bien que souvent très longs, ils sont connus de tous et se présentent comme une "justification à postériori d'un partage inégal de l'autorité" pour reprendre la définition de R. Bastide (7). Ils sont donc bien cette "charte sociale" dont parlait B. Malinowski, et ils fournissent les arguments garantissant la forme existante de la société.

Les mythes de peuplement expliquent pourquoi, alors qu'il existe plusieurs clans d'origines diverses représentes sur chaque massif, un seul, toujours le même, fournit le chef de massif ou de quartier. A chaque fois, la domination de ce clan s'imposant aux autres est liée à la possession des pierres de pluie, qui viennent renforcer son pouvoir et finalement en apparaissent comme le symbole.

Deux schémas de peuplement se dégagent. Dans le premier, on trouve la présence d'autochtones, rejoints par plusieurs vagues d'imigrants, et supplantés par eux, les premiers arrivants fournissant un chef au nouvel ensemble formé. (On remarquera qu'il n'existe pas chez les Mofu de cas ou les autochtones assimilent les envahisseurs et conservent la chefferie).

(7) R. Bastide: Formes élémentaires de la Stratification sociale, Paris. C.D.U. 1965 ct. p. 24. 
Dans le second schéma, le massif est présenté comme vide. Ou bien, comme les autochtones, les premiers arrivés sont évincés du pouvoir par ceux qui les rejoignent, ou bien ces nouveaux immigrants acceptent leur autorité.

Dans tous ces cas, les pierres de pluie apparaissent en filigrane de la constitution d'une autorité centrale.

a) présence d'autochtones.

Deux hypothèses sont possibles :

- les pierres de pluie peuvent avoir été trouvées sur place par le premier clan ayant rejoint les autochtones.

C'est ce qu'on observe dans le massif de Wazan, occupe autrefois par des autochtones très primitifs, les Siler. Les Siler, dit le mythe, ne construisaient pas de cases et vivaient dans des grottes. Ils étaient vêtus de feuilles et c'est aussi avec des feuilles qu'ils enterraient leurs morts ; ils ne connaissaient pas le mil et vivaient de cueillette; enfin ils $n$ 'avaient pas de pierres de pluie. Les nouveaux arrivants, les Erkeţ̧e, qui s'imposent à eux grâce à leurs richesses, en particulier grâce à leur sel, $n$ 'en avaient pas non plus. Mais, une fois leur supériorité reconnue, ils se mettent à en trouver et le chef Erketรe devient faiseur de pluie.

- les pierres peuvent aussi avoir été apportées par le clan venu rejoindre les autochtones qui, là aussi, subissent la loi du nouvel arrivant.

C'est le cas dans le massif de Durum, peuple d'abord par deux clans autochtones, les Fogom et les MokuZek. Les informateurs le soulignent : aucun n'avait de pierres de pluie. Un vieux Mokužek m'a dit: "Parmi les deux clans, il n'y avait personne qui pouvait faire venir la pluie. Donc on ne pouvait pas dire qu'il y avait vraiment un chef".Puis, arrive le clan Mandzah, venu du sud-est, qui apporte avec lui ses pierres de pluie. "Lui pouvait faire pleuvoir : il était donc chef". En fait, cette reconnaissance des Mandzah comme clan dirigeant a été longue et s'est faite par l'élimination des autochtones, les Mokuzek par le feu, les Fogom par l'eau. Le mythe montre comment grâce à leurs pierres de pluie, les Mandzah ont fait tomber tant de pluie qu'ils en ont rempli un barrage où ils ont noyé les Fogom par ruse.

De la même façon à Dugwor, les immigrants Dingiže, venus du sud-est avec des pierres de pluie, s'imposent comme chefs à des autochtones, les Wulger, aussi "sauvages" que les Siler de Wazan (grottes, cueillette, etc...)

On notera que dans tous ces mythes de peuplement ou 
apparaissent des autochtones, ceux-ci ignorent totalement $1^{\prime u}$ sage des pierres de pluie.

b) massifs vides.

- les pierres de pluie peuvent avoir été apportées ou trouvées par les premiers arrivants qui en ont ensuite été dépouillés par le clan fournissant aujourd'hui le chef.

Telle est la situation sur le massif de Duvangar, où les Laway, clan dominant actuellement, reconnaît avoir trouvé à son arrivée sur le massif, deux clans déjà installés, car immigrés à date plus ancienne, chacun dans son quartier, chacun avec ses pierres de pluie, les Mowayan et les Sidaway. Les informateurs disent: "Chaque clan avait ses pierres de pluie, donc chacun était chef chez soi".

Les Laway s'installent aux côtés des Sidaway et il $y$ a d'abord une période de coexistence durant laquelle les Laway se multiplient et vivent en symbiose avec les Sidaway dont ils reconnaissent l'autorité. Puis les Laway s'attaquent aux Sidaway et les contraignent à leur abandonner à la fois la chefferie et les pierres de pluie, d'une façon assez curieuse. Le chef Sidaway de l'époque - auquel étaient encore soumis les Laway, devenus pourtant plus nombreux et plus riches - était appelé Bi-Džuley, "chef stérile", car il n'avait pas d'enfant. De sonvivant, il avait attaché autour de sa jambre un kwalay, recouvert de feuilles comme s'il s'agissait d'un pansement protégeant une plaie, et il avait recommandé de ne pas l'enlever. A sa mort, on enterra Bi-Džuley avec son "pansement", personne ne soupçonnant ce qu'il cachait. Peu de temps après, une grande sêcheresse survint et le devin découvrit que le responsable en était Bi-Džuley. Ses dires furent confirmés par des enfants qui racontèrent avoir vu Bi-Džuley avant sa mort cacher une pierre contre sa plaie. Le devin ordonna alors de sacrifier un taureau et de déterrer Bi-Džuley. Or, les Sidaway étaient trop pauvre pour offrir un taureau. Ce furent les Laway qui le fournirent, faisant tous les sacrifices prescrits sur la tombe de Bi-Dźuley qu'ils réenterrèrent suivant les ordres du devin. Bi-Džuley, devenu lui-même kwalay, passa aux mains des Laway qui construisirent leurs cases à l'endroit où étaient les anciens chefs Sidaway, tout en haut de la montagne.

En même temps, les Laway s'attaquaient aux Mowayan, installés à mi-pente du massif dans un quartier séparé. Les Laway entretenaient avec eux des relations d'amitié. Un jour, un Laway veut faire de la bière et donc faire sécher son mil germé. Mais il pleut trop. Il s'en désole devant le Mowayan qui lui prête "une chose", son kwalay; la pluie s'arrête et le mil sèche. Le Laway décide alors de voler son kwalay au Mowayan. Lorsqu'a son tour le Mowayan veut faire sécher son mil germé en saison des pluies, on assiste à une inversion des rôles. Le Laway sort le kwalay volé, arrête la pluie et dit: "Tu vois ! Tu dis que tu es chef, mais c'est moi qui ai arrêté la pluie !" Le Mowayan, dépossédé, 
doit reconnaftre la superiorite du Laway.

Lorsque d'autres clans arrivent ensuite sur le massif, ils trouvent un seul clan capable de faire pleuvoir, le clan Laway dont dépendent désormais Sidaway et Mowayan. Les nouveaux arrivants reconnaissent à leur tour les Laway pour chefs.

Ce thème du "vol du kwalay", que l'on pourrait appeler encore le thème de "l'ami pervers", se retrouve sur divers massifs.

Ainsi, à Molkwo, on voit un kwalay prêté par amitié par le clan Maya au clan Mukyo, puis arraché par la violence, son ancien possesseur trop complaisant étant assassiné par l'ancêtre du chef actuel. Du coup, un troisième clan possédant des "pierres à pluie", pris de peur, en fait don au même ancêtre, car elies ne lui servent plus à rien, dit-il.

- les pierres de pluie ont été apportées par le premier clan installé dont l'autorité a été reconnue par les clans arrivés ensuite.

Ainsi à Mikiri, le clan du chef, le clan Mandza, remonte à un frère cadet d'un chef du massif de Durum. A la suite d'une dispute, le cadet aurait quitté Durum - il y a 8 générations - en volant au chef, son frère ainé, une partie de ses pierres de pluie qu'il aurait ensuite complétées par des découvertes faites sur Mikiri, son nouveau massif. Les quatre clans installés par la suite à Mik:́ri n'essayèrent pas. de contester l'autoritê du clan Mandza fondateur, fournissant toujours le chef aujourd'hui.

- dernière modalité de cette liaison entre chefferie et pierres de pluie : le clan arrivé le premier est obligé par ceux qui l'ont rejoint, d'accepter les pierres de pluie qu'ils ont découvertes.

Makabay, montagne habitée puis désertée au XIXe siècle lors des razzias peul, a téé recolonisée tout récemment, après la grande famine de 1930. Le premier Mofu à s'y installer avec sa famille s'est vu apporter à plusieurs reprises des pierres de pluie que ses voisins l'ont forcé à prendre "pour que je fasse pleuvoir", m'a-t-il dit.

c) partage de la chefferie.

Le lien entre pouvoir de pierres etpluie est si bien établi que lorsqu'un groupe éclate, il y a partage des pierres de pluie. 
Sur le massif géographique de Durum, coexistent aujourd'hui deux"massifs" sociologiques, deux chefferies indépendantes, ayant toutes deux à leur tête le clan Mandzah. En effet, après avoir chassé les autochtones, les Mandzah étaient devenus tròs nombreux. Ils furent contraints à la division par l'habilité d'un cadet qui, à la mort de son père, coucha avec les femmes de celui-ci. Toutes furent enceintes. Les anciens fort ennuyés, décidèrent que le cadet succéderait à son père, contrairement à toutes les habitudes mofu ; quant à l'ainé, Mangerdla, ils l'envoyèrent fonder sa propre chefferie à l'autre extrémité du massif de Durum, en lui donnant la moitié des pierres de pluie. Cette chefferie porte aujourd'hui son nom. "Va habiter la-bas maintenant. Tu as ton kwalay; tu peux arrêter les pluies. Tu as tes bizi yam; tu peux faire venir les pluies. Et les gens de là-bas pourront travailler pour toi".

De même, sur le massif de Dugwor, le clan du chef, le clan Dingiže, se sépara en deux chefferies jumelles, installées de part et d'autre du massif, après avoir divisé en. deux parts égales les pierres de pluie.

Tous ces exemples mettent en valeur le lien entre chefferie et pierres de pluie. Un vrai chef doit avoir des pierres de pluie; pas de pierres de pluie, pas de chefferie. Inversement, posséder des pierres de pluie,c'est être chef. Leur possession est le couronnement de l'autorité.

Ces récits présentant 1'acquisition de ces pierres de pluie comme un fait progressif invitent à se demander s'il $y$ a eu un foyer d'où serait parti ce trait culturel : la liaison entre chefferie et pierre de pluie. Si on s'en tient aux seuls massifs Mofu, on trouve deux directions indiquées : l'est ou le sud-est des massifs actuels qui est la direction d'origine des clans possédant la chefferie(Laway à Duvangar, Mandzah à Durum, Erketše à Wazan, Dingiže à Dugwor) parfois le nord (Maya et Mukuno à Molkwo). L'ouest n'est jamais indiqué, bien que divers clans en soient originaires.

\section{3) Manifestations de ce pouvoir sur la pluie.}

Sans que cela soit dit expressément par les informateurs, on peut considérer que le pouvoir du chef sur la pluie a deux faces :

- une face licite, se manisfestant au grand jour par I'accomplissement des rites pour demander ou parfois pour diminuer la pluie. 
- une face illicite que l'on aperçoit en parlant avec les membres du clan. En cas de secheresse prolongee, les chefs sont toujours soupçonnes de l'avoir sciemment provoquee, par un ressentiment quelconque.

\section{a) le pouvoir licite.}

- les rites de demande de pluie.

Les Mofu n'imaginent pas que les pluies sont liees de façon absolue au pouvoir du chef. Ils constatent que les premières pluies viennent de toutes façons et toujours de 1 'Est, appelé parfols pour cette raison "le coin de l'eau". Mais, après la. ou les toutes premières pluies qui, en gros, tombent fin avrildébut mai, s'étend une période de six semaines environ - de la mi-mai a la fin-juin - avant la saison des pluies proprement dite, durant laquelle il pleut très peu, alors que le mil est déjà sorti de terre. C'est à ce moment là surtout qu'on attend des chefs qu'ils manifestent leur pouvoir sur la pluie et qu'ils fassent pleuvoir à intervalles réguliers pour que le mil puisse croitre.

Chaque chef fait necessairement chaque annee, a date fixe, un sacrifice avec ses bizi yam, ses "enfants de la pluie". Là, il compte les lunes, depuis la lune de la récolte du mil de montagne, qui marque le début de l'annee, jusqu'a la lune des semailles, normalement la 6ème. Il procède alors a son sacrifice. S'il ne pleut pas ou pas assez, il renouvelle son sacrifice, cette fois sans plus s'occuper de la lune, "sinon le mil secherałt". Chaque anné, un chef de pluie fait donc un nombre de sacrifices variable, pouvant aller jusqu'a 6 ou 7 .

La régularité annuelle de ces sacrifices est indispensable. Il s'agit pour le chef de montrer que c'est lui qui amène la pluie. Il doit donc aussi les faire tôt. s'il attend, et que la pluie tombe, alors que les gens de son massif savent qu'il n'a pas célébré son sacrifice, ils conclueront que leur chef ne sert a rien. C'est ce que chuchotaient en mai 1969 les habitants de Duvangar. La pluie etait venue plusieurs fois, or le chef n'avait pas encore procéde à son sacrifice. 
Par contre, si le chef fait son sacrifice et que la saison des pluies sulvante est abondante et régulière, les gens diront qu'ils ont un bon chef, "a qui les "enfants de la plule" obêissent bien". C'est donc d'après le volume des pluies tombant sous son règne qu'un chef sera jugé bon ou mauvais.

\section{Schéma du sacrifice.}

(Je prendrai l'exemple d'un sacrifice exécuté en ma présence a Wazan en mai 1969).

Le sacrifice mangat $y_{i}$ bizi yam, "enduire les enfants de la pluie", commence très tôt le matin. Une chèvre est égorgée et offerte aux ancêtres-hommes du chef de massif, ceux de la salle des greniers et celui - plus éloigné qui est gardien de la porte. On invoque ces ancêtres:" Nous sommes venus demander la pluie ! Qu'elle vienne ! Mon père, il faut que la pluie vienne sans danger !". Pendant ce temps, les pierres ont été sorties de leur pot. Elles sont lavées avec de l'eau puis enduites de graisse de boa.

"Si on mettait simplement de l'eau sur les pierres, la pluie viendrait mais accompagnée de tonnerre, risquant de tuer quelqu'un. Tandis qu'avec la graisse, la pluie viendra doucement, comme le serpent qui se coule et se glisse dans les herbes". On lave à nouveau les pierres avec de l'eau dans laquelle sont mélangées des feuilles de jujubier et de la sève gluante de 1'arbuste lewer. Enfin, les pierres sont enduites du sang de la chèvre et du contenu de son intestin, zikotof, toujours pour éviter le tonnerre.

Puis, on offre à l'extérieur un poulet au génie de la montagne, le mbolom Arkay que dessert le chef de massif. "Voila, tu es le mbolom Arkay. Nous voulons la pluie. Nous offrons ce sacrifice pour demander la plvie. Qu'il n'y ait pas de foudre et que la pluie tombe aujourd'hui même! Prends ce poulet et fais tomber la pluie !". Enfin, on rentre dans la salle des greniers ou l'on fait une offrande tous les ancêtres, même les plus éloignés, avec de la bière et de la viande grillée.

Un autre sacrifice de mangatši bizi yam auquel j'ai assisté en juin 1970 sur le "massif" de Morley, présenta le même schéma avec seulement des invocations plus longues: "Pardon mon père, pardon Dieu, Je vous en supplie! Il est temps maintenant que la pluie vienne. Nous vous offrons ce sacrifice comme avant. Autrefois à cette époque-ci il y avait déjà des pluies ! S'il n'y a pas de pluie, nous allons tous mourir..."

L'assistance aux sacrifices permet de préciser à qui s'adressent ces sacrifices. Ils ne sont jamais offerts aux bizi yam mais, collectivement, aux ancêtres, babaay, aux esprits de la montagne, mbolom, et à Dieu, Bi-Erlam.Ainsi que me le disait Marlamtsa, forge ron-devin de Wazan, en 1973, "quand 


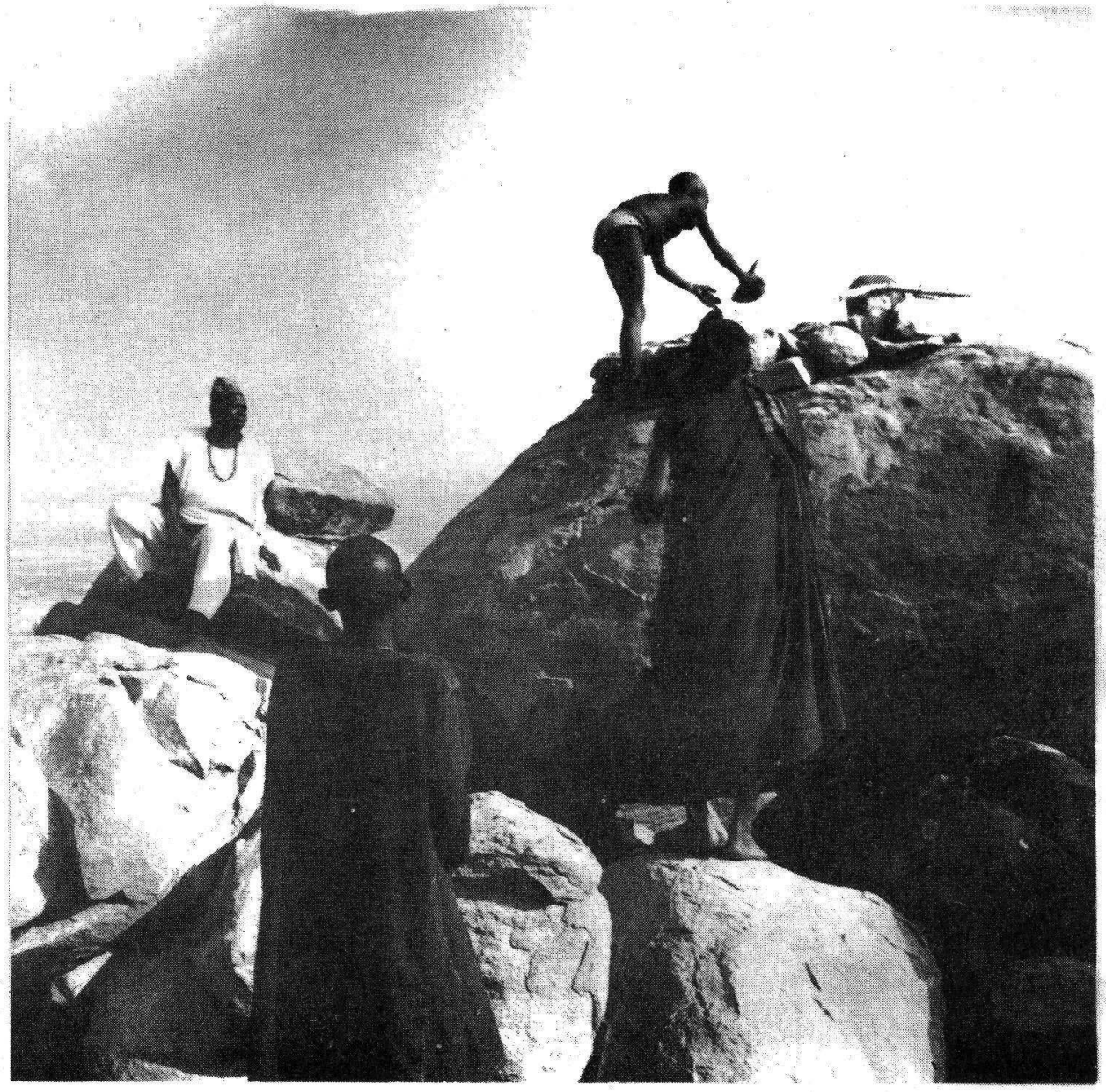

Offrande de bière durant un sacrifice au mbolom, génie de la montagne Arkay (Nord-Cameroun) 
on fait le sacrifice aux "enfants de la pluie", on le fait pour les ancêtres, mais Dieu aide tout. Tout marche par aide de Dieu. S'il y a madama (impureté), les ancêtres et Dieu sont fâchés. Les ancêtres le rendent malade, et Dieu arrête les pluies, mais les ancêtres, eux, ne peuvent pas tout seuls arrêter les pluies".

- les rites d'arrêt des pluies.

Aucun chef ne déclare provoquer sciemment une sécheresse prolongée, interrompant de façon anormale la saison des pluies. Tout ce qu'il revendique, c'est une sécheresse limitée au début de la saison des pluies - pour laisser aux gens de son massif le temps: de semer par exemple - ou à la fin de la saison des pluies - pour que le mil, mis à sécher dans les champs, ne soit pas mouillé par une pluie intempestive qui risquerait de le faire pourrir. Ainsi, en octobre 1968, un des chefs du massif de Dugwor me déclarait-il avoir empêché la pluie de tomber grâce à ses rites. Des motifs personnels peuvent aussi intervenir. Le chef de Mokyo, à Molkwa, procède, selon ses propres dires, aux rites d'arrêt des pluies, lorsqu'en saison des pluies il veut non aller au marché de Dogba, à $4 \mathrm{~km}$ de chez lui, sans risquer d'être mouillé...

Les rites d'arrêt de la pluie - auxquels je n'ai jamais assisté et que je me suis seulement fait decrire sont simples. Le chef prend une paille ou une plume trempée dans l'ocre rouge et touche avec elle l'endroit où se trouve le kwalay. Puis il la plante dans son toit ; tant qu'il ne l'enlèvera pas, il ne pleuvra pas.

b) le pouvoir illicite.

En cas de sécheresse persistante et inopportune, lorsque le mil commence à se flettrir, deux explications sont possibles.

Dieu, les ancêtres et les génies de la montagne, les mbolom, peuvent être contrariés à cause d'un acte d'impureté, un madama, commis sur le massif. En plus, mécaniquement, le madama s'accumule et forme au-dessus du massif une barrière empêchant les pluies de tomber. Le remède consiste en un sacrifice aux ancêtres, avec manipulation des "enfants de la pluie", 
suivant le schéma indiqué plus haut. Mais ce sacrifice doit être précédé d'une purification de tout le massif.

Il existe une autre explication : l'action d'un chef de massif qui arrête les pluies pour des motifs personnels. En 1973, les Mofu autour de Meri disaient qu'il pleuvait mal parce que la jeune fille sur qui le chef de massif de Meri avait des vues comme huitième femme, avait préféré épouser un jeune homme de son âge. D'où le courroux du prétendant évincé.

Sur un massif donné, les habitants soupçonneront soit leur propre chef, soit un chef voisin dont le pouvoir dépasserait ainsi les limites de son terroir. Beaucoup plus rarement, on incrimine l'action d'un détenteur caché de kwalay. Pour faire cesser la sécheresse, on organise des supplications publiques avec intimidation du chef concerné.

Entreprendre cette démarche c'est, pour les Mofu, "pleurer la pluie" matui yam. Elle se fait quartier par quartier et prend naissance spontanément, sans décision du chef de quartier ou du devin, à l'initiative d'un homme quelconque. "Celui qui revient de son champ et qui pleure beaucoup parce que trop de mil est en train de secher sur pied dans sa plantation prend sa flûte". Il peut être imité de ses voisins et, le lendemain, tous les hommes du quartier partent de très bonne heure, habillés en guerriers avec leur casque, leur bouclier, leur arc et leur carquois de flèches. Ils ont aussi leur flûte. Sous la direction d'un devin, ils se rendent chez le chef de massif en faisant des détours pour s'approcher des grands arbres où le kwalay a pu se réfugier. Toute la journée, ils jouent de la flûte et se livrent à des pantomimes guerrières.

En 1973, à Wazan, 4 quartiers sur 6 sont allés "pleurer la pluie" chez le chef de Wazan. Il s'agit d'une véritable démonstration de force au cours de laquelle on interpelle le chef brutalement, au mépris des règles habituelles de respect. "Est-ce toi le responsable ?" Jamais, font remarquer les informateurs, un chef ne réponara "oui, c'est moi" ! II calme les gens en leur disant "Non, ce n'est pas moi ! Moi aussi je pleure !" La promesse d'un sacrifice spécial les incite à retourner chez eux. 
Toujours en 1973, I'un de ces quatre quartiers, limitrophe du chef de Morley, est alle ensuite "pleurer la pluie" chez lui. Les gens ont cassé toutes les poteries du chef de Morley et renverse l'eau qu'ils ont trouvée. "Autrefois en allant chez un chef etranger, on pouvait tuer un des hommes recontrés".

Le sacrifice spécial offert par le chef se fait à la fois sur les bizi yam et sur le kwalay, pour le cas, dit le chef, où le kwalay serait sorti tout seul, à son insu, tandis que les gens, eux, pensent, "pour faire rentrer le kwalay, mis sciemment à l'extérieur par le chef lui-même". Beaucoup sont persuadés que si on battait ou, mieux, si on tuait le chef, il pleuvrait aussitôt.

Il existe donc une diffërence essentielle entre chef de pluie mofu et roi sacré. C'est la personne même du roi sacré qui est liée à la prospérité du pays. Une sécheresse traduit la mauvaise santé du roi qui n'est que responsable indirect des calamités naturelles. Pour les Mofu au contraire, une sécheresse est dûe le plus souvent à un acte d'irritation consciente du chef qui, heureusement, peut défaire ce qu'il avait commencé et rendre la pluie.

\section{Conclusion :}

Ce lien privilégié entre chef et pluie n'est pas particulier aux Mofu. On le retrouve chez les Giziga, leurs voisins immédiats de l'est, et, un peu plus à l'est, chez les Mundang qui tiennent leur chef pour responsable de la pluie. Encore plus à l'est, ce sont les zaghawa qui demandent à leur chef d'offrir les sacrifices appropriés qui leur vaudront la pluie.

Le chef Mofu, lui aussi, apparait comme le médiateur qui utilise la longue lignée de ses ancêtres pour réclamer la pluie. Mais il ne se contente pas de ce rôle, modeste, somme toute, de demandeur, d'interprète. Il est aussi celui qui obtient la pluie. "Nous avons un bon chef", dit avec satisfaction le massif mofu qui a connu une saison des pluies régulière. Cette affirmation du pouvoir du chef sur les éléments apparaft comme la traduction symbolique de son emprise sur le groupe 
- $161-$

humain qui lui est soumis. En même temps, elle rêvêle le besoin qu'ont les Mofu de croire qu'il existe un ordre dans la nature et que cet ordre est subordonne à l'homme. 


\section{DISCUSSION}

L. de HEUSCH - Vous faites allusion an sacrifice exceptionnel en l'honneur du kwalay lorsque le chef a abusé de son pouvoir. Avez-vous des renseignements sur ce sacrifice specifique qui aurait pour objet d'arrêter l'action maléfique du kwalay?

J.F. VINCENT - Dans ce cas, on offre une chèvre rouge; c'est tout ce que je sais.

L. de HEUSCH - Dans cette dichotomie, il y aurait une conception différente des genies attachés aux pierres : dans un cas, vous avez des génies mortels assimilés à des poissons; dans l'autre, un personnage qui ne meurt pas, et qu'on doit enfermer soigneusement. D'autre part, l'association symbolique du kwalay et de la couleur rouge avec l'arc en ciel se retrouve en Afrique centrale.

G. DIETERLEN - Je me demande si ce qui reste d'autorité au Moro Naba ne tient pas uniquement à son pouvoir sur la pluie. De même, le Hogon d'Arou, qui n'a plus aucune autorite politique, reste le maitre de la pluie et de la sécheresse.

J.F. VINCENT - En relisant le livre de J. Rouch sur les songhay, $j$ 'ai trouvê aussi cet appel à un rituel avec des pierres de pluie.

J. ROUCH - Chez les Songhay, l'arc en ciel est considéré d'une façon qui n'est pas néfaste parce que c'est lui qui met l'eau dans les puits. Il est responsable des eaux souterraines. C'estprobablement aussi le cas de votre "arrêteur de pluie".

L. de HEUSCH. - Oui, c'est vrai aussi en Afrique centrale.

J. ROVCH - L'arc en clel est un serpent multicolore qui arrête avec son dos la pluie dans les nuages et la met dans la terre.

N. ECHARD - Chez les Hausa, il est le gardien dans les puits de l'eau souterraine. Dans les puits, il ne boit pas d'eau. nuand la pluie apparait dans le ciel, il sort pour boire l'eau des nuages, mais il ne la met pas dans le puits.

J. ROUCH

- Nous venons de trouver dans un site archeologique en face de Niamey une quantite de ces oeufs de pierre pour lesquels nous 
n'avons aucune explication. Ils sont très petits : on avait pense que c'etaient des concretions calcaires mais on les a trouvés dans le sol à côté de haches et d'objets néolithiques, authentiques ceux-la, car les Songhay fabriquent de faux objets neolithiques pour les mettre sur leurs autels... On ignore encore la signification de ces pierres.

- Pour moi, les boules de pierres mofu sont des raviveurs à mortier très usés. Un raviveur a mortier qui a servi pendant une quinzaine d'années perd ses angles et prend la forme d'une boule. Cependant, si chez les songhay, ces pierres rondes sont si petites, ce ne peut être cela...

G. DIETEREEN - Je voudrais dire quelque chose sur le symbolisme des pierres. Vous avez bien démontré que chez les Mofu ces pierres n'étaient pas des esprits auxquels on adressait des prières, qu'elles étaient des intermédiaires ou des symboles de quelque chose.Or, c'est exactement cette même position que l'on peut observer chez les Bambara et chez les Dogon. Mais, il est possible d'aller plus loin, car les Dogon et les Barbara ont expliqué ce qu'elles représentent pour eux : ce sont des éléments bioloqiques. Les pierres taillées ou roulées, les haches néolithiques, ne sont pas des esprits. Cependant, elles sont vivantes, car on les considère comme les éléments minéraux de la semence masculine du géniteur mythique de l'humanité ; l'eau de pluie est l'eau de cette même semence.

J.F. VINCENT - Je n'ai traité qu'une petite partie du problème. Il y a des groupes mofu qui ont des maitres de la pluie ayant très peu d'importance et je pense que dans les autres oroupes, l'jmportance qui leur est donnée est due à des influences extérieures. - Vous croyez ? Il existe en effet des spécialistes faiseurs de pluie ambulants.

G. $L E M O A L$ - Il faut voir aussi ce qui se passe dans les sociétés sans chef.

R. VERDIER - Quel genre de flute utilisent les Mofu ?

J.F. VINCENT - Ce sont des flutes d'hommes ayant chacune une seule note, haute. ou grave. Lorsque ces flûtes jouent ensemble un air donné, il $\mathrm{y}$ a au début une certaine cacophonie, puis les flûtes s'accordent. 
M. CARTRY

- Ainsi le chef mofu n'arrête la pluie que par intérêt personnel ?

J.F. VINCENT

- Oui. Mais, ce n'est pas lui qui arréte la pluie quand il y a eu madama, acte d'impureté. La pluie s'arrête alors par la volonté des ancetres, des esprits de la montaọne ou du Dieu suprême, irrités par cette impureté.

M. CARTRY

- L'accusation d'arrêt de pluie portée contre un chef est-elle faite à l'instigation du devin ?

J.F. VINCENT

- Non. Elle est portée spontanément par les gens du massif concerné. Il n'y a pas non plus de décision prise en commun au niveau des chefs de quartiers. 